\title{
Efecto de edulcorantes no calóricos en la aceptabilidad sensorial de un producto horneado.
}

\section{Effect of non-caloric sweeteners in sensory acceptability of a baked product}

\begin{abstract}
Samples of cakes (baked biscuit-type products) made with sugar (control) and samples prepared with non-caloric sweeteners (stevia, saccharin and sucralose) were developed and acceptability evaluated using a 5-point hedonic scale test for color, aroma and flavor. The color and aroma results indicated that the cakes made with sugar have significantly larger acceptability than cakes made with non-caloric sweeteners $(p<0.05)$. In assessing the acceptability of taste there are significant differences between all samples of cakes, with the following descending order according to the sweetener used: sugar> stevia> sucralose> saccharin. Replacement of sugar by non-caloric sweeteners in cakes, decreased the acceptability of the products. One explanation may be because no non-enzymatic browning reactions occur such as Maillard reaction and caramelization, which generate compounds related to color, aroma and flavor of baked foods.
\end{abstract}

Key words: sugar, non-caloric sweeteners, sensory reactions, Maillard reaction, caramelization

\section{INTRODUCCIÓN}

La obesidad presenta una tendencia creciente a nivel mundial, tanto en países desarrollados como en vías de desarrollo. De acuerdo a la OMS, es uno de los problemas más importante en el mundo por las graves consecuencias para la salud a corto y largo plazo. El sobrepeso y la obesidad constituyen factores de riesgo para el desarrollo de patologías crónicas entre los más jóvenes como la hipertensión arterial, diabetes mellitus tipo 2, colelitiasis, algunos tipos de cáncer, entre otras ${ }^{1}$.

La dieta, actividad física y factores genéticos contribuyen a la obesidad en niños y adultos, a pesar de ello, los hábitos alimenticios parecen determinar el mayor impacto en la ganancia rápida de peso, como por ejemplo el consumo de azúcar y alimentos con alta concentración de ésta ${ }^{2}$.

El consumo excesivo de azúcar ha sido un tema en salud pública, por su posible rol en desplazar o diluir nutrientes
Vilma Quitral', Ma. Angélica González², Constanza Carrera3 ${ }^{3}$ Gabriela Gallo ${ }^{3}$, Paola Moyano ${ }^{3}$, Julieta Salinas ${ }^{3}$, Paula Jiménez ${ }^{3}$.

1. Escuela de Nutrición y Dietética. Facultad de Salud. Universidad Santo Tomás

2. Escuela de Nutrición y Dietética. Facultad de Ciencias de la Salud. Universidad de Talca 3. Departamento de Nutrición. Facultad de Medicina. Universidad de Chile.

Dirigir correspondencia a: Prof. Vilma Quitral. Escuela de Nutrición y Dietética. Facultad de Salud. Universidad Santo Tomás. Avenida Ejército 146. Santiago. Teléfono 223624956. Email: vilmaquitral@santotomas.cl

Este trabajo fue recibido el 21 de marzo de 2016 y aceptado para ser publicado el 23 de diciembre de 2016.

en la dieta y contribuir a la epidemia de la obesidad. Sin embargo, los estudios sobre efectos del consumo de azúcar en el aumento de peso han mostrado resultados contradictorios. Existen publicaciones que indican que el azúcar es la principal fuente de ingesta de energía en los niños y adolescentes, además el consumo de bebidas endulzadas con azúcar se asocia con la obesidad infantil². Algunos investigadores sostienen que el reemplazo de azúcar por edulcorantes no calóricos facilitaría la pérdida de peso ${ }^{3-5}$ particularmente en corto tiempo, otras investigaciones han encontrado una asociación entre el consumo de edulcorantes no calóricos y ganancia de peso, principalmente a largo plazo $^{6,7}$. Además se ha encontrado una asociación positiva entre el consumo de edulcorantes no calóricos con síndrome metabólico y diabetes tipo $2^{8-10}$; y se asocia al aumento del riesgo de intolerancia a la glucosa, debido a cambios en la composición y función de la microbiota ${ }^{11,12}$. 
Existen en la actualidad numerosos edulcorantes no calóricos en el mercado que se utilizan para reemplazar el azúcar. Sin embargo, el azúcar no solo aporta sabor dulce a los alimentos, es uno de los ingredientes con mayor funcionalidad en los productos horneados, por lo que es muy difícil encontrar un sustituto capaz de realizar sus mismas funciones. El azúcar es un agente que ayuda a estabilizar la humedad en el alimento y limitar el hinchamiento del almidón, lo cual se traduce en una textura más fina del producto final ${ }^{13}$.

De hecho, la presencia de azúcar aumenta la temperatura de gelatinización del almidón y de la desnaturalización proteica, así como la viscosidad de la masa. También, ayuda a promover la agregación de cristales de grasa y, por lo tanto, mejora la captación de aire durante el batido y la estabilización durante el horneado. Por último, mejora la microestructura, la porosidad y el volumen del producto final y contribuye al pardeamiento u oscurecimiento, tanto de la corteza como de la miga del alimento ${ }^{13}$.

Para poder establecer el reemplazo de edulcorantes no calóricos por azúcar en alimentos, se debe comprobar la calidad sensorial de éstos. La aceptabilidad sensorial de los edulcorantes depende de la similitud que tengan con el azúcar ${ }^{14}$. Para establecer si los consumidores reconocen diferencias entre productos elaborados con azúcar y elaborados con edulcorantes no calóricos, y si éstos mejoran el grado de satisfacción del mismo, se utilizan pruebas de aceptabilidad en las que los consumidores indican el grado de satisfacción de cada producto ${ }^{15}$.

El objetivo del presente estudio es comparar la aceptabilidad sensorial de un producto horneado elaborado con azúcar (control), con muestras elaboradas con edulcorantes no calóricos (estevia, sucralosa y sacarina). Para esto se elaboraron queques, que son productos horneados tipo bizcocho con azúcar y con diferentes edulcorantes no calóricos.

\section{MATERIAL Y MÉTODOS}

Se elaboraron queques de acuerdo a una receta tradicional: $140 \mathrm{~g}$ de harina, $30 \mathrm{ml}$ de aceite, 1 huevo, 100 $\mathrm{ml}$ de leche, 6 g de polvos de hornear y 120 g de azúcar, se mezclaron todos los ingredientes, y se formó una masa fluida homogénea, se vertió en un molde metálico y se llevó a $180{ }^{\circ} \mathrm{C}$ en horno por 40 minutos.

Se seleccionaron edulcorantes no calóricos disponibles en el mercado: estevia, sacarina y sucralosa, en tabletas y en polvo aptos para hornear. Estos edulcorantes se adicionaron a los queques en reemplazo del azúcar, en cantidades equivalentes de dulzor: $4,8 \mathrm{~g}$ de estevia; $1,32 \mathrm{~g}$ de sacarina o 0,192 g de sucralosa. Se mantuvieron las concentraciones de los demás ingredientes, las condiciones de elaboración, tiempo y temperatura de horneo.

Para la evaluación de la aceptabilidad sensorial, se escogieron estudiantes universitarios de 18 a 29 años de edad, que consumen azúcar o edulcorantes habitualmente y productos que los contienen; los criterios de exclusión fueron estudiantes con diabetes mellitus tipo 1 o 2 , y estudiantes que no consumían ningún alimento con azúcar.

La determinación del tamaño de la muestra para la prueba de aceptabilidad, se basó en el estudio de Hough y et.al. ${ }^{16}$, considerando una probabilidad de error tipo I $(\alpha)$ de $1 \%$, probabilidad de error tipo II (ß) de $5 \%$, RMSL (error cuadrado medio dividido por la longitud de escala) de 0,30 y diferencia en las medias que se busca en el experimento de 0,2 , lo que dio como resultado un tamaño de muestra de 59 individuos, por lo que se trabajó con 60 individuos.

Los evaluadores fueron citados en diferentes días, durante la mañana o tarde (en horas intermedias entre desayuno o almuerzo), en cada cita se evaluó solo una muestra. La distribución de los queques para cada evaluador se realizó al azar, así como la numeración de los platos que contenían las muestras. Las evaluaciones sensoriales se realizaron en un laboratorio que cuenta con cabinas individuales aisladas y condiciones apropiadas para este propósito ${ }^{15,17}$. El esquema de la intervención se presenta en la figura 1 . Se realizaron 3 repeticiones del ensayo.

Para la evaluación sensorial se utilizó una prueba de aceptabilidad con escala hedónica de 5 puntos por parámetros, que califica desde 1 "me disgusta mucho" a 5 "me gusta mucho"18, evaluando 3 parámetros: color, aroma y sabor; como se presenta en la figura 2.

Los resultados se analizaron por ANOVA y prueba de Duncan para determinar diferencias significativas con nivel de significación de 5\%. Se utilizó el programa SPSS 15.0.

\section{RESULTADOS}

La figura 3 presenta la evaluación de la aceptabilidad de las muestras de queque en cuanto al color. Se observa que no existen diferencias significativas entre los queques endulzados con estevia, sacarina y sucralosa como edulcorante, presentando una puntuación entre "no me gusta ni me disgusta" y "me gusta". La muestra control (queque endulzado con azúcar) presenta puntuación significativamente mayor que las otras muestras $(p<0,05)$.

En la figura 4 se observa que al utilizar como edulcorante estevia, sacarina o sucralosa, no existen diferencias significativas en la aceptabilidad del aroma de los queques, la muestra control endulzada con azúcar presenta aceptabilidad del aroma significativamente mayor $(p<0,05)$.

La figura 5 demuestra que existen diferencias significativas en el sabor de los queques que se endulzaron con estevia, sacarina, sucralosa y azúcar $(p<0,05)$. La menor aceptabilidad corresponde a los queques endulzados con sacarina, y la mayor aceptabilidad a los queques endulzados con azúcar.

\section{DISCUSIÓN}

El color de los queques preparados con azúcar presentó mejor evaluación ya que fue dorado brillante, típico de alimentos horneados, mientras que los queques preparados con edulcorantes no calóricos, presentaron un color pálido y sin brillo. Estos resultados coinciden con lo que reportan Manisha y et.al. ${ }^{19}$, quienes reemplazaron azúcar 
en queques por estevia y sorbitol líquido, y encontraron que los queques preparados con azúcar presentaron una corteza de color café dorado.

El color de la superficie es una característica muy importante en productos horneados, porque contribuye a definir la preferencia del consumidor. El color de la superficie depende de características fisicoquímicas de la masa (como por ejemplo contenido de agua, pH, azúcares reductores y aminoácidos) y de las condiciones de proceso que se aplican (temperatura, humedad relativa, transferencia térmica, etc.). El color de la superficie de los alimentos horneados es un punto crítico, ya que desde el punto de vista tecnológico, es uno de los objetivos del proceso de horneado $^{20}$. Las reacciones químicas que provocan el color característico de alimentos horneados corresponden a pardeamiento no enzimático que incluye reacciones de Maillard y de caramelización ${ }^{21}$.

La reacción de Maillard involucra una serie de reacciones que comienzan cuando azúcares reductores, principalmente D-glucosa, reaccionan con un grupo amino libre de un aminoácido que es parte de una cadena de proteína ${ }^{21,22}$.

La caramelización es otra reacción de pardeamiento no enzimático que afecta a los azúcares al someterlos a alta temperatura. Generalmente ocurre en forma simultánea con la reacción de Maillard ${ }^{23,24}$. Por esta razón los queques endulzados con edulcorantes no calóricos no presentan el color dorado ya que al no estar presente el azúcar no hay sustratos para la reacción de pardeamiento no enzimático.

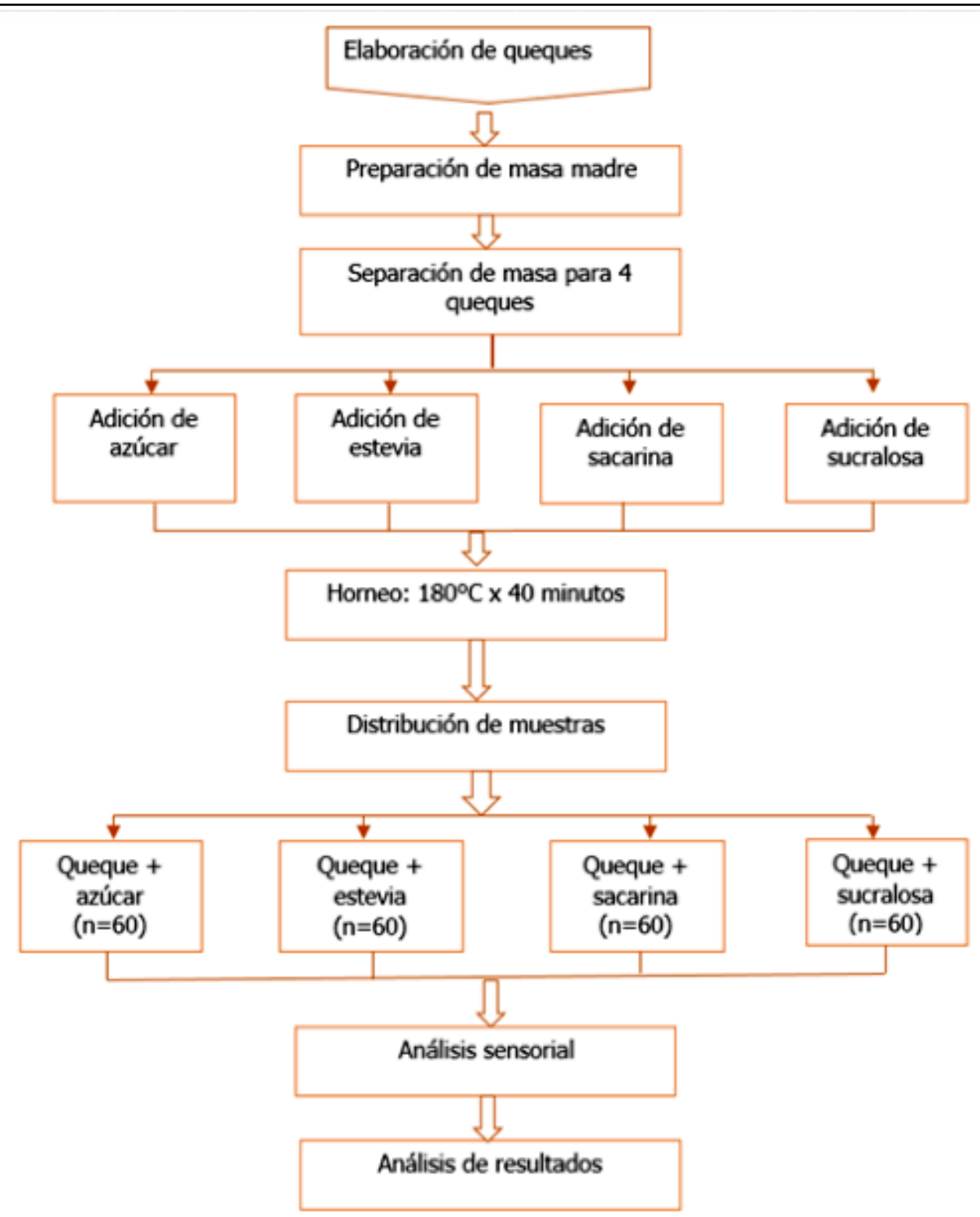

Figura 1. Esquema de la intervención. 
De acuerdo a la Escala hedónica de 5 puntos, califique los parámetros ed color, aroma y sabor de las muestras que se presenta:

\begin{tabular}{|l|c|}
\hline \multicolumn{2}{|c|}{ Escala hedónica } \\
\hline Categoria & número \\
\hline Me gusta mucho & 5 \\
\hline Me gusta & 4 \\
\hline No me gusta ni me disgusta & 3 \\
\hline Me disgusta & 2 \\
\hline Me disgusta mucho & 1 \\
\hline
\end{tabular}

\begin{tabular}{|l|l|l|l|}
\hline Muestra & Color & Aroma & Sabor \\
\hline & & & \\
\hline
\end{tabular}

Comentarios:

Figura 2. Ejemplo de la ficha de respuesta para la evaluación de aceptabilidad de queques.

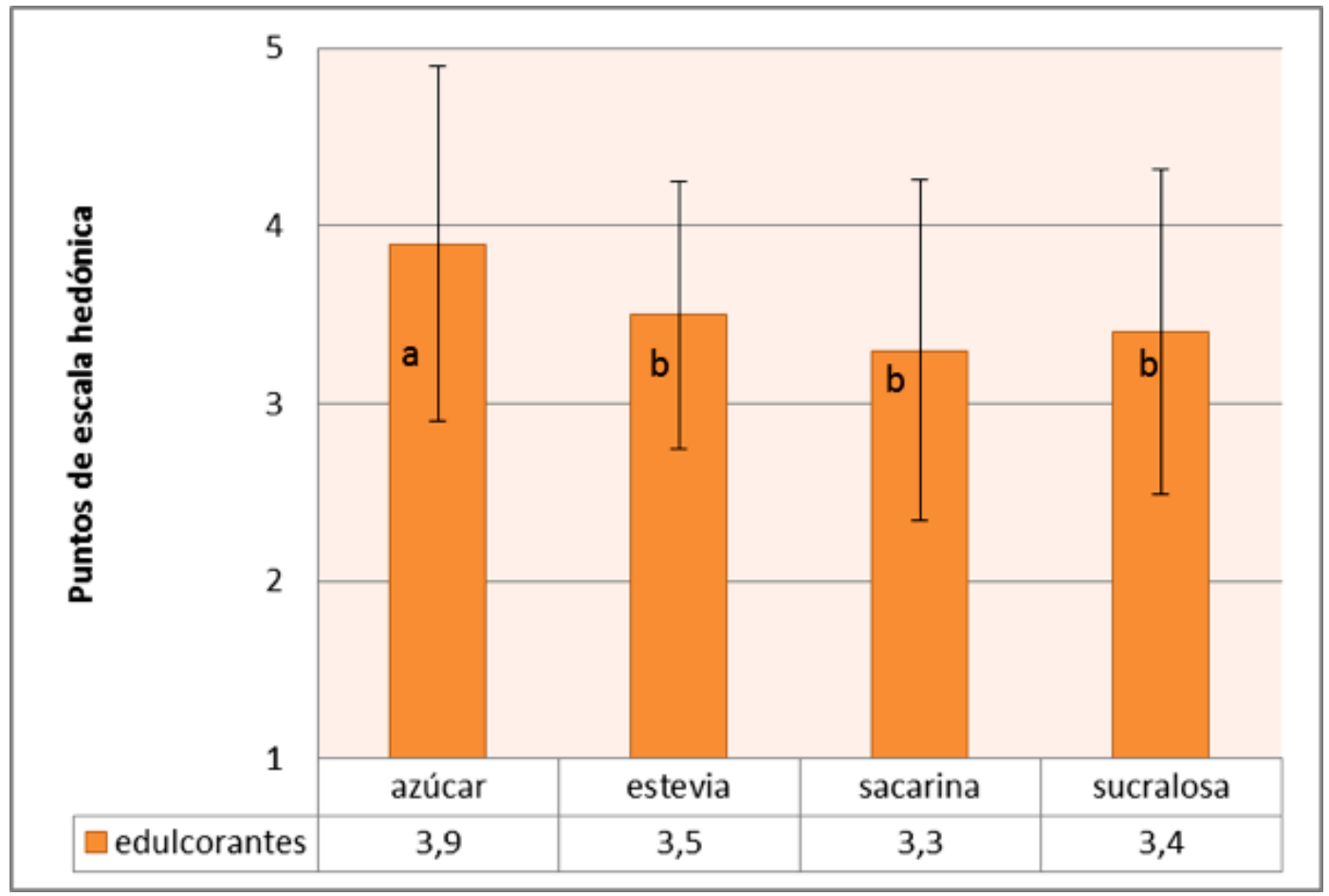

Figura 3. Aceptabilidad del color de queques adicionados de diferentes edulcorantes. Letras distintas indican diferencias estadísticamente significativas (Duncan, $\mathrm{p}<0,05$ ) 


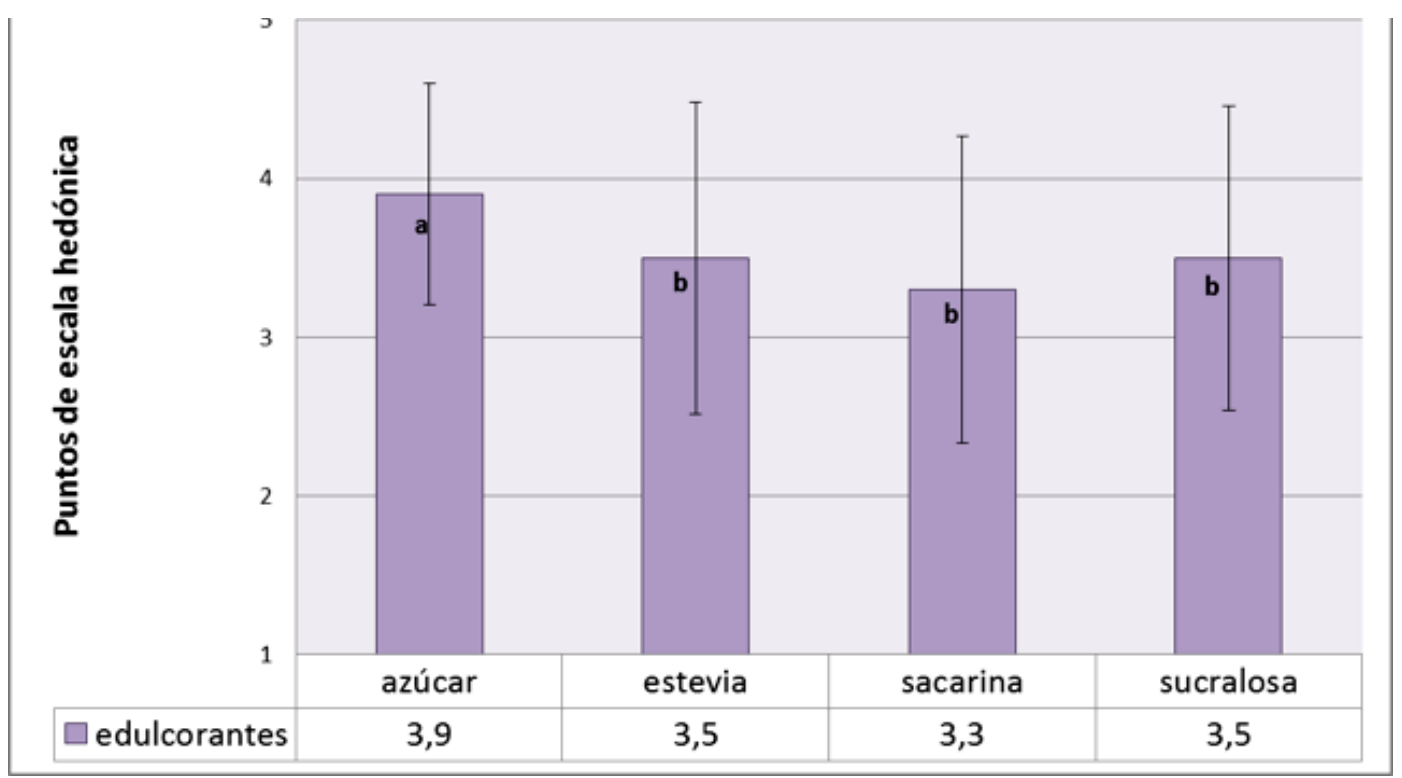

Figura 4. Aceptabilidad del aroma de queques adicionados de diferentes edulcorantes Letras distintas indican diferencias estadísticamente significativas (Duncan, $p<0,05$ )

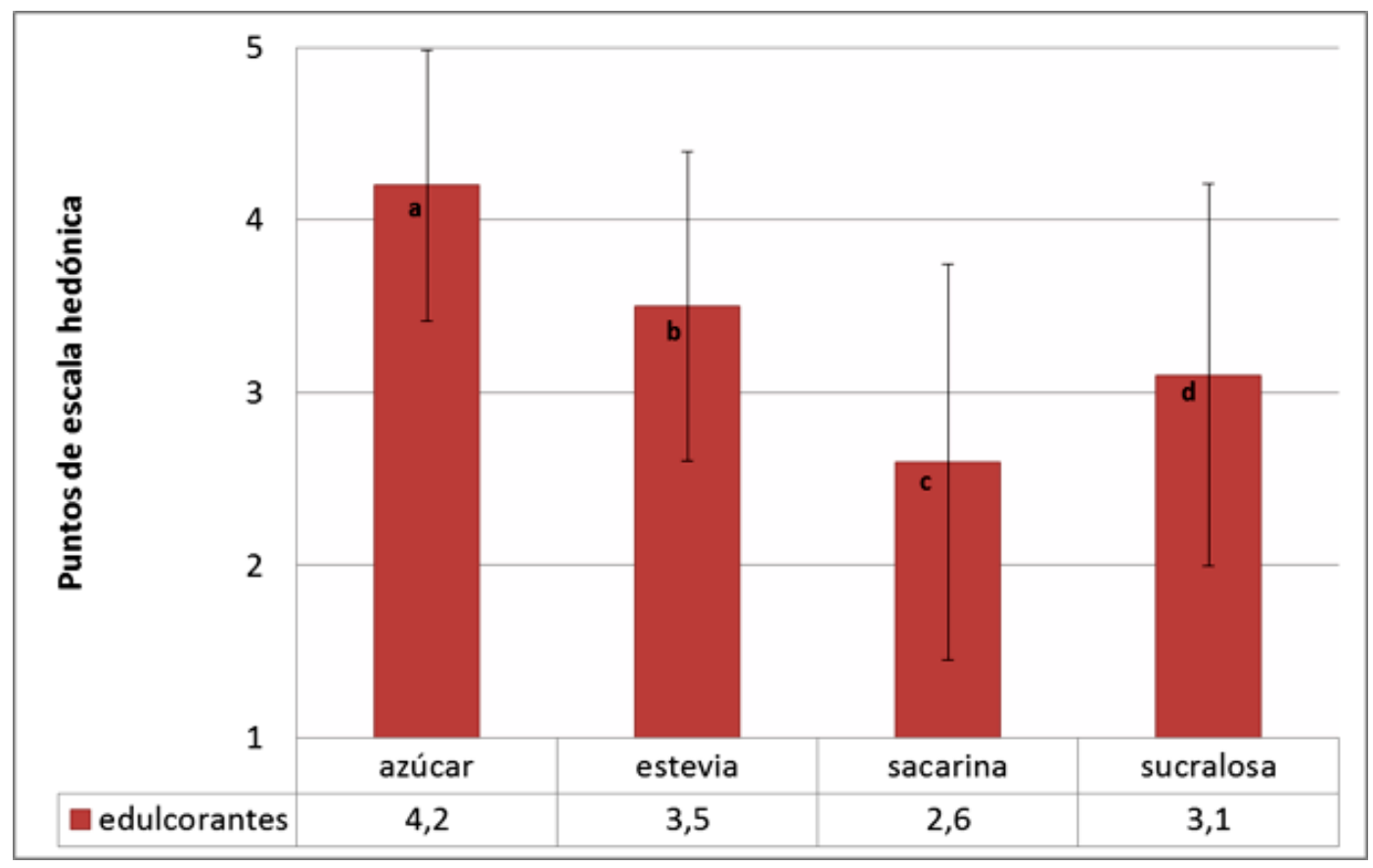

Figura 5. Aceptabilidad del sabor de queques adicionados de diferentes edulcorantes. Letras distintas indican diferencias estadísticamente significativas (Duncan, $\mathrm{p}<0,05$ )

El reemplazo de azúcar en la elaboración de queques afecta el color, tanto de la corteza como de la miga, así lo comprobaron Manisha y et.al. ${ }^{19}$, evaluando el color por método instrumental. La corteza se hace más clara y amarilla, mientras que la miga se hace más oscura. La evaluación sensorial de los queques indicó que la muestra control (elaborada con azúcar) mostró un color café dorado en la corteza, la forma de la corteza fue la mejor evaluada en comparación con las muestras con edulcorantes ya que presentó una forma de cúpula y más alta; el color de la miga fue blanco crema, el grano de la miga fue fino, textura suave y sensación agradable en la boca. 
La mayor aceptabilidad de aroma y sabor obtenida por los queques con azúcar también se debe a la las reacciones de Maillard y caramelización. Resultados que coinciden con Attia y et.al. ${ }^{25}$ que trabajaron con bizcocho.

Las reacciones de pardeamiento no enzimático producen compuestos que, aparte de contribuir al desarrollo de color característico, también contribuyen en el sabor y aroma de los alimentos horneados, lo cual es de gran importancia para la aceptabilidad por parte de los consumidores. En la reacción de Maillard, el tipo de compuestos que aportan sabor dependen del tipo de azúcar y aminoácidos involucrados en la reacción, además de temperatura, tiempo, $\mathrm{pH}$ y contenido de agua del sistema los que influyen en la cinética de la reacción ${ }^{24,26,27}$. En general, el primer factor (tipo de azúcar y aminoácidos) determinan el tipo de compuesto formado relacionado con el sabor. El segundo factor, referido a las condiciones de la reacción, influencia la cinética de ésta. Por ejemplo si el aminoácido prolina participa en la reacción de Maillard, el sabor que se desarrolla es el típico de pan, arroz, palomitas de maíz. Otros productos de la reacción de Maillard también contribuyen al sabor y aroma como las pirazinas (cereales tostados), alquilpiridinas (amargo, astringente, quemado como en café, cebada y malta), furanos, furanonas, piranonas (caramelo), etc. ${ }^{27}$.

Martínez-Cervera y et.al. ${ }^{28}$, reemplazaron sacarosa en un producto horneado, total o parcialmente $(25 \%, 50 \%$ y $75 \%)$ por una mezcla de sucralosa (polidextrosa). El reemplazo de sacarosa afectó las propiedades reológicas de la masa, disminuyendo la viscosidad, viscoelasticidad y gravedad específica, lo que provocó que los productos presentaran menos altura, elasticidad, cohesividad, masticabilidad y resilencia y un menor número de células de aire que el control. A pesar de ello, los autores determinaron que al reemplazar la sacarosa en un $50 \%$ por la mezcla de sucralosa, la apariencia, color, textura, sabor, dulzor y aceptabilidad general fue similar a la muestra control (100\% sacarosa). La muestra con $100 \%$ de reemplazo de sacarosa tuvo una aceptabilidad significativamente menor que el control.

Por otro lado, Martínez-Cervera y et.al. ${ }^{29}$, reemplazaron sacarosa por polioles, no encontrándose diferencias significativas en la aceptabilidad sensorial.

Guggisberg y et.al. ${ }^{30}$, endulzaron muestras de yogurt con sacarosa $(8 \mathrm{~g} / 100 \mathrm{~g})$ y estevia en diferentes concentraciones $(0,04 ; 0,035 ; 0,03$ y $0,025 \mathrm{~g} / 100 \mathrm{~g})$ y evaluaron características sensoriales mediante el método de análisis descriptivo. Los resultados demostraron que las muestras con azúcar presentaron mayor sabor dulce, menor acidez y menor amargor en forma significativa respecto a las muestras endulzadas con estevia; por otro lado, se presentó un sabor extraño en los yogurts con estevia, lo que aumentaba a mayores concentraciones del edulcorante.

Respecto al endulzante estevia, De Souza y et.al. ${ }^{14}$, la consideran como el mejor endulzante no calórico, porque reduce el sabor amargo que otorgan otros edulcorantes, mientras que otros autores sostienen que estevia presenta sabor amargo, especialmente en altas concentraciones ${ }^{31}$.
Morais y et.al..$^{32}$, desarrollaron un postre de chocolate endulzado con azúcar y edulcorantes no calóricos, evaluaron la aceptabilidad sensorial con escala hedónica de 9 puntos y los resultados revelaron que el dulzor ideal se logró con 8,13\% de azúcar, seguido de sucralosa, aspartame y estevia.

En chocolates con leche no existieron diferencias en los atributos de brillo, aroma a cacao, aroma a manteca de cacao y sabor cacao, entre muestras con azúcar y muestras con sucralosa y estevia, al aplicar una prueba de análisis cuantitativo descriptivo con jueces entrenados. Al realizar prueba de aceptabilidad con 116 consumidores, se aplicó una escala lineal no estructurada de 9 puntos, encontrando que la apariencia de los chocolates era similar en todas las muestras, sin embargo, la muestra con azúcar superaba significativamente la evaluación de aroma, sabor y textura respecto a las muestras con sucralosa y estevia. En el caso de sabor, la muestra con estevia fue significativamente peor evaluada que la muestra con sucralosa ${ }^{33}$.

De Souza y et.al ${ }^{14}$, compararon edulcorantes no calóricos (sucralosa, acesulfame-K, taumatina, glucósido de esteviol) con azúcar en mermeladas de frutas y determinaron que los edulcorantes estudiados produjeron alta percepción de sabor amargo, la duración total de la percepción del sabor amargo fue alta. Sin embargo, los autores sostienen que sucralosa tiene un perfil de sabor muy similar al de azúcar, presenta además bajo nivel de amargor y acidez. Por otra parte, Mendonça y et.al ${ }^{34}$, elaboraron mermeladas de durazno reemplazando azúcar por sucralosa, acesulfame-k y mezcla de ambos edulcorantes. En el análisis sensorial se observó un sabor metálico residual en las mermeladas con acesulfame- $K$ y la combinación de [sucralosa + acesulfame-K]. Los autores concluyeron que las mermeladas con sucralosa presentaron similitud con las mermeladas con azúcar en cuanto a atributos sensoriales, además fueron estables durante el almacenamiento del producto.

\section{CONCLUSIÓN}

El reemplazo de azúcar por edulcorantes no calóricos en queque, perjudica el color, aroma y sabor de los productos, debido a que con azúcar se producen reacciones de pardeamiento no enzimáticos en el alimento horneado que generan compuestos relacionados que mejoran el color, aroma y sabor de los queques.

\section{RESUMEN}

Se elaboraron muestras de queques (productos horneados tipo bizcocho) con azúcar (control) y con edulcorantes no calóricos (estevia, sacarina y sucralosa). Los queques se evaluaron sensorialmente mediante una prueba de aceptabilidad con una escala hedónica de 5 puntos para los parámetros de color, aroma y sabor. Los resultados de color y aroma indican que los queques elaborados con azúcar tienen una aceptabilidad significativamente mayor que los queques elaborados con los edulcorantes no calóricos $(p<0,05)$. En la evaluación de sabor hubo diferencias significativas entre todas las muestras de queques, con el siguiente orden decreciente según el 
edulcorante utilizado: azúcar > estevia > sucralosa > sacarina. El reemplazo de azúcar por edulcorantes no calóricos en los queque, disminuyó la aceptabilidad de los productos. Una explicación posible es que no se producen reacciones de pardeamiento no enzimático como reacción de Maillard y caramelización, las que generan compuestos relacionados con el color, aroma y sabor de productos horneados.

Palabras clave: azúcar, edulcorantes no calóricos, sensorial, reacción de Maillard, caramelización.

\section{BIBLIOGRAFÍA}

1. Aguilar M. Gonzalez E. Garcia C.Garcia L. Alvarez J. Padilla C. Mur N. Comparative study of the effectiveness of body mass index and the body-fat percentage as methods for the diagnosis of overweight and obesity in children. Nutr Hosp 2012; 27: 185-191.

2. Song WO. Wang Y. Chung CE. Song B. Lee W. Chun OK. Is obesity development associated with dietary sugar intake in the U.S? Nutrition 2012; 28: 1137-1141.

3. Raben A. Vasilaras TH. Moller A. Astrup A. Sucrose compared with artificial sweeteners. Different effects on ad libitum food intake and body weight after 10 weeks of supplementation in overweight subjets. Am J Clin Nutr 2002; 76: 721-729.

4. Mattes R. Popkin B. Nonnutritive sweetener consumption in humans: effects on appetite and food intake and their putative mechanism. Am / Clin Nutr 2009; 89: 1-4.

5. De Ruyter J. Olthof M. Seidell J. Katan M. A trial of sugar-free or sugar-sweetened beverages and body weight in children. New Engl I Med 2012; 367: 1397-1406.

6. Fowler SP. Williams K. Resendez R. Hunt K. Hazuda H. Stern M. Fueling the obesity epidemic? Artificially sweetened beverage use and long-term weight gain. Obesity 2008; 16: 1894-1900.

7. Nettleton J. Lutsey P. Wang Y. Lima J. Michos E. Jacobs D. Diet soda intake and risk of incident metabolic syndrome and type 2 diabetes in the multi-ethnic study of atherosclerosis (MESA). Diabetes Care 2009; 32: 688-694.

8. Lutsey P. Steffen L. Stevens J. Dietary intake and the development of the metabolic syndrome: the atherosclerosis risk in communities study. Circulation 2008; 117: 754-761.

9. De Koning L.Malik V. Rimm E. Willet W. Hu F. Sugar-sweetened and artificially sweetened beverage consumption and risk of type 2 diabetes in men. Am J Clin Nutr 2011; 93: 1321-1327.

10. Swithers $S$. Artificial sweeteners produce the counterintuitive effect of inducing metabolic derangements. Trends Endocrin Met 2013; 24: 431-441.

11. Suez J. Korem T. Zeevi D. Zilberman-Schapira G. Thaiss C. Maza O. Israeli D. Zmora N. Gilad S. Weinberger A. Kuperman Y. Harmelin A. Kolodkin-Gal I. Shapiro H. Halpern Z. Segal E. Elinav E. Artificial sweeteners induce g/ucose intolerance by altering the gut microbiota. Nature 2014; 514: 181-186.

12. Burke M. Small D. Physiological mechanism by which nonnutritive sweeteners may impact body weight and metabolism. Physiol Behav 2015; 152: 381-388.

13. Beesley PM. Sugar functionality reviewed. Food Technol Int Eu 1995; 87-89.

14. De Souza V. Pereira P. Pinheiro A. Bolini H. Borges S. Queiroz $F$. Analysis of various sweeteners in low-sugar mixed fruit jam: equivalent sweetness, time-intensity analysis and acceptance test. Int J Food Sci Technol 2013; 48: 1541-1548.

15. Carpenter R. Lyon D. Hasdell T. Análisis Sensorial en el
Desarrollo y Control de la Calidad de los Alimentos. Editorial Acribia. Zaragoza, España. 2000.

16. Hough G. Wakeling I. Mucci A. Chambers E. Mendez I. Rangel L. Number of consumers necessary for sensory acceptability tests. Food Qual Prefer 2006; 17: 522-526.

17. Norma UNE-EN ISO 8589:2010. Análisis sensorial. Guía general para el diseño de una sala de cata. 2010.

18. Guinard IX. Sensory and consumer testing with children. Trends Food Sci Tech 2001; 11: 273-283.

19. Manisha G. Soumya C. Indrani D. Studies on interaction between stevioside, liquid sorbitol, hydrocolloids and emulsifiers for replacement of sugar in cakes. Food Hydrocolloids 2012; 29: 363-373.

20. Zanoni B. Peri C. Bruno D. Modelling of browning kinetics of bread crust during baking. Food Sci Technol-Leb 1995; 28 : 604-609.

21. Virág D. Kiss A. Forgó P. Csutorás C. Molnár S. Study on Maillard-reaction driven transformations and increase of antioxidant activity in lysine fortified biscuits. Microchem J 2013; 107: 172-177.

22. Kim J-S. Lee Y-S. Effect on reaction $\mathrm{pH}$ on enolization and racemization reactions of glucose and fructose on heating with amino acid enantiomers and formation of melanoidins as result of the Maillard reaction. Food Chem. 2008;108:582-592.

23. Fennema O. R. Química de los Alimentos. Editorial Acribia. Zaragoza. España. 2005.

24. Purlis E. Browning development in bakery products - A review. J Food Eng 2010; 99: 239-249.

25. Attia EA. Shehata HA. Askar A. An alternative formula for the sweetening of reduced-calorie cakes. Food Chem 1993; 48: 169-172.

26. Martins S. Jongen W. Van Boekel M. A review of Maillard reaction in food and implications to kinetic modeling. Trends Food Sci Tech 2001; 11: 364-373.

27. Van Boekel MA. Formation of flavour compounds in the Maillard reaction. Biotechnol Adv 2006; 24: 230-233.

28. Martínez-Cervera S. Sanz T. Salvador A. Fiszman SM. Rheological, textural and sensorial properties of low-sucrose muffins reformulated with sucralose/polydextrose. Food Sci Technol-Leb 2012; 45: 213-220.

29. Martínez-Cervera S. Salvador A. Sanz T. Comparison of different polyols as total sucrose replacers in muffins: Thermal, rheological, texture and acceptability properties. Food Hydrocolloids 2014; $35: 1-8$.

30. Guggisberg D. Piccinali P. Schreier K. Effects of sugar substitution with Stevia Actiligth ${ }^{T M}$ and Stevia combinations or Palatinose ${ }^{T M}$ on rheological and sensory characteristics of low-fat and whole milk set yoghurt. Int Dairy J 2011; 21: 636-644.

31. Cardoso J. Bolini H. Descriptive profile of peach nectar sweetened with sucrose and different sweeteners. I Sens Stud 2008; 23: 804-816.

32. Morais EC. Morais AR. Cruz AG. Bolini H. Development of chocolate dairy dessert with addition of prebiotics and replacement of sucrose with different high-intensity sweeteners. J Dairy Sci 2014; 97: 2600-2609.

33. Medeiros de Melo L. Bolini H. Efraim P. Sensory profile, acceptability, and their relationship for diabetic/reduced calorie chocolates. Food Qual Prefer 2009; 20: 138-143.

34. Mendonça C. Zambiazi R. Gularte M. Granada G. Caracteristicas sensoriais de compotas de pêssego light elaboradas com sucralose e acesulfame-K. Ciênc Tecnol Alimnent Campinas 2005; 25: 401-407. 